\section{Miastenia gravis refractaria con buena respuesta a rituximab. Caso clínico}

\author{
FELIPE SUÁREZ H. ${ }^{1,2}$, DANIELA URRUTIA E. ${ }^{1,2}$
}

\section{Refractory myasthenia gravis with good response to rituximab. Report of one case}

Myasthenia gravis (MG) is a heterogeneous disease, and there is no unique therapeutic approach for all patients. In 2013 the Myasthenia Gravis American Foundation (MGFA) panel of experts defined refractory $M G$ as the lack of change or deterioration after the use of corticosteroids and two immunosuppressive agents, in adequate doses and time. We report a 51-years-old female with MG of bulbar predominance, who presented four myasthenic crises in 17 months despite the use of corticosteroids, azathioprine and mycophenolate. The high costs associated with her hospitalizations, as well as severe caloric - protein malnutrition, the need for tracheostomy and gastrostomy support, led us to use rituximab. The patient evolved with an excellent response, free of crises after 30 months. She gained $12 \mathrm{~kg}$ of weight, without tracheostomy and gastrostomy, only using pyridostigmine support 4 times a day.

(Rev Med Chile 2020; 148: 1031-1033)

Key words: Biological Therapy; Myasthenia Gravis; Rituximab.
'Departamento de Neurología,

Hospital Base Osorno. Osorno,

Chile.

2Universidad Austral de Chile.

Osorno, Chile.

Trabajo no recibió financiamiento.

Los autores declaran no tener

conflictos de interés.

Recibido el 24 de agosto de

2019, aceptado el 24 de mayo de 2020.

Correspondencia a:

Dr. Felipe Suárez H.

Guillermo Buhler 1765, Osorno,

Chile.

felisuarezh@gmail.com
I a miastenia gratis (MG) es una enfermedad adquirida de la unión neuromuscular, que resulta de la unión de anticuerpos a los componentes de la unión neuromuscular, más frecuentemente a los AChR -Ab. Otros anticuerpos relacionados con la MG son la kinasa músculo-específica (MuSK) y la proteína relacionada contra el receptor de lipoproteína 4 (LRP4). La incidencia varia de $0,3-2,8$ por 100.000 habitantes universalmente ${ }^{1}$. En Chile, la prevalencia en el sudeste de Santiago se ha calculado en 8,36 por 100.000 habitantes $^{2}$.

La característica clínica más relevante es la fatigabilidad, la cual se expresa como una debilidad que suele ser simétrica y que aumenta con el ejercicio y el uso repetitivo del músculo ${ }^{3}$. El pronóstico, la calidad de vida y la sobrevida en general son buenas. La terapia está enfocada en la remisión farmacológica total o casi total ${ }^{3}$.

En octubre de 2013 la Fundación Americana de MG (MGFA) reunió a un panel de 15 expertos en MG para desarrollar un consenso respecto a la enfermedad. Se definió MG refractaria cuando el estado post intervención no cambió o empeoró tras el uso de corticoides y al menos 2 agentes inmunosupresores, en dosis y tiempo adecuados, o hubo efectos colaterales de los fármacos que limitaron su uso, definido por el paciente y su médico ${ }^{1}$. Se estima que la MG refractaria afecta de 10 al 15\% del total de pacientes con $\mathrm{MG}^{4}$.

Debido a que la MG es heterogénea no existe una aproximación terapéutica universal para todos los pacientes. Además la heterogenicidad de los estudios randomizados tienen generalizaciones limitadas y los estudios no controlados son limitados por el potencial sesgo ${ }^{1}$.

El uso de terapias biológicas ha surgido como un arma terapéutica relevante que promete tener un efecto más significativo como ahorrador de corticoides, comparado con las terapias estándar 
e incluso inducir remisión ${ }^{4}$. El rituximab es un anticuerpo monoclonal quimérico anti-CD20 que ha demostrado efectividad en varias series de casos clínicos no controlados, así como también en dos metaanálisis y en una revisión sistemática ${ }^{5,6}$.

\section{Caso clínico}

Mujer de 51 años, diagnosticada con MG de predominio bulbar en 1981, sin estudio de anticuerpos. En 1991 fue timectomizada. Dentro de sus fármacos de uso habitual, destacaban: piridostigmina $60 \mathrm{mg}$ cuatro veces al día, prednisona en dosis de 5 a $20 \mathrm{mg}$ al día, y azatioprina $100 \mathrm{mg}$ al día.

En agosto y diciembre de 2015 presentó 2 crisis miasténicas gatilladas por neumonía, por lo que requirió ventilación mecánica invasiva e inmunoglobulina, en dosis de $0,4 \mathrm{~g} / \mathrm{Kg} /$ día por 5 días. Posterior a la segunda crisis miasténica y en el contexto de desnutrición calórico-proteica severa, con un índice de masa corporal de 13,7 y trastorno deglutorio severo, se instaló traqueostomía y gastrostomía. Se cambió de azatioprina a mofetil micofenolato $500 \mathrm{mg} \mathrm{c} / 12 \mathrm{~h}$. En agosto de 2016 y enero de 2017 presenta respectivamente la tercera y cuarta crisis miasténica, ambas gatilladas por neumonía, por lo que requiere nuevamente ventilación mecánica invasiva y uso de inmunoglobulina en la dosis antes indicada.

Considerando la ocurrencia de cuatro crisis miasténicas en 17 meses, con falta de respuesta a dos inmunosupresores en dosis adecuadas, se cataloga como MG refractaria, lo cual sumado al predominio bulbar de sus manifestaciones y sospecha de MG subtipo anti-MuSK, hizo planteable iniciar rituximab en dosis de $375 \mathrm{mg} / \mathrm{m}^{2}$ semanal por 4 semanas cada 6 meses.

Seis meses después de la primera dosis de rituximab, la paciente logra una resolución completa de su fatigabilidad. Se baja prednisona y micofenolato lentamente hasta suspensión y queda con apoyo de piridostigmina $60 \mathrm{mg}$ por 4 veces al día. Debido a buena evolución se retiró gastrostomía y traqueostomía. Durante el año 2018 se decide mantener con dos pulsos de rituximab separados por 6 meses, completando 4 pulsos en total, suspendiendo posteriormente las infusiones. Actualmente la paciente se ha mantenido sin descompensaciones por 30 meses y presentó aumento de 12 kilos de peso desde la última crisis.

Se obtuvo consentimiento informado de la paciente para la publicación.

\section{Discusión}

A la fecha no existe un consenso de un algoritmo terapéutico ideal para la MG, como tampoco la comparación head-to-head de las distintas opciones terapéuticas. El resultado final común es lograr la remisión clínica con el régimen menos tóxico y más tolerable posible ${ }^{4}$.

Un inmunosupresor no esteroidal debería ser adicionado a un corticoide cuando los efectos colaterales de los corticoides son significativos, cuando la respuesta a corticoides es inadecuada o cuando los corticoides no se pueden reducir debido a recurrencia de síntomas al bajarlos ${ }^{1}$.

Respecto al tipo de inmunosupresor no esteroidal existe una amplia variación en la práctica clínica, debido a que solo hay escasa literatura que respalde la recomendación de alguno de ellos en particular. El consenso de expertos y algunos ensayos randomizados avalan el uso de azatioprina como agente inmunosupresor de primera línea ${ }^{1}$.

La timectomía ha demostrado ser efectiva en pacientes con MG generalizada, estables, con menos de 5 años de evolución, edad menor a 65 años, con AChR-Ab positivos. Ésta es una cirugía electiva donde se prefiere el uso de videoasistencia o cirugía robótica ${ }^{7}$. La evidencia no avala la indicación de timectomia en paciente con anticuerpos MuSK, LRP4 o agrina ${ }^{4}$.

El uso de terapias biológicas ha surgido como un arma terapéutica relevante que promete tener un efecto más significativo como ahorrador de corticoide que las terapias estándar e incluso inducir remisión ${ }^{4}$.

Dos metanálisis y una revisión sistemática avalan el uso de rituximab en MG refractaria, siendo más efectiva en pacientes con anticuperos MuSK que en pacientes con anticuerpos AChR-Ab. El régimen más usado de rituximab fue $375 \mathrm{mg} / \mathrm{m}^{2}$ semanal por 4 semanas $s^{5,6}$.

El tratamiento con rituximab es efectivo en el $50-70 \%$ de los pacientes con MG. En un estudio de resultados a 10 años plazo, demostró que el tratamiento con rituximab es seguro, logra una 
eficacia clínica sostenida, permite la disminución de terapias inmunosupresoras, reduce los costos hospitalarios e impacta en el potencial de maternidad en forma notable ${ }^{4}$.

La necesidad de dosis de mantención en respondedores sigue siendo empírica. Algunos expertos prefieren repetir la infusión cuando ocurre una recurrencia, mientras otros administran $2 \mathrm{~g}$ cada 6 meses o $1 \mathrm{~g}$ cada 3 meses hasta asegurar estabilidad ${ }^{4}$. Existe evidencia clase IV de que el régimen de $375 \mathrm{mg} / \mathrm{m}^{2}$ semanal por 4 semanas, luego mensualmente por 2 meses, logra menores tasas de recurrencia y una respuesta más duradera ${ }^{7}$.

\section{Conclusiones}

Si bien no existen algoritmos terapéuticos ideales para la MG, los casos de MG refractaria abren la posibilidad del uso de agentes biológicos monoclonales, dentro de los cuales el uso de rituximab ha sido docuemntado en series de casos, dos revisiones sistméticas y un metaanálisis. El tratamiento con rituximab en pacientes bien seleccionados, permite una eficacia clínica sostenida e incluso remisiones, la reducción de agentes inmunosupresores y disminuye los costos hospitalarios asociados a las crisis miasténicas.

\section{Referencias}

1. Sanders D, Wolfe G, Evoli A, Gilhus N, Illa I, Kuntz N, et al. International consensus guidance for management of myasthenia gravis: excecutive summary. Neurology 2016; 87: 419-25.

2. Cea G, Martínez D, Salinas R, Vidal C, Hoffmeister L, Stuardo A. Clinical and epidemiological features of myasthenia gravis in Chilean population. Acta Neurol Scand 2018; 138 (4): 338-43.

3. Gilhus N. Myasthenia Gravis. N Engl J Med 2016; 375: 2570-81

4. Dalakas M. Immunotherapy in myasthenia gravis in the era of biologics. Nat Rev Neurol 2019; 15 (2): 113 24.

5. Iorio R, Damato V, Alboini P, Evoli A. Efficacy and safety of rituximab for myasthenia gravis: a systematic review and meta-analysis. J Neurol 2015; 262 (5): 11159.

6. Tandan R, Hehir MK, Waheed W, Howard DB. Rituximab treatment of myasthenia gravis: a systematic review. Muscle Nerve 2017; 56 (2): 185-96.

7. Wolfe G, Kaminski I, Aban G, Minisman H, Kuo C, Marx A, et al. Randomized trial of thymectomy in myasthenia gravis. N Engl J Med 2016; 375: 511-22.

8. Cortés-Vicente E, Rojas-García R, Díaz-Manera J, Querol L, Casasnovas C, Guerrero-Sola A, et al. The impact of rituximab infusion protocol on the long-term outcome in anti-MuSK myasthenia gravis. Ann Clin Transl Neurol 2018; 5 (6): 710-6. 